\title{
Adiabatic chemical freeze-out and wide resonance modification in a thermal medium
}

\author{
K.A. Bugaev* \\ Bogolyubov Institute for Theoretical Physics, Metrologichna str. 14 ${ }^{B}$, Kiev 03680, Ukraine \\ E-mail: Bugaev@th.physik.uni-frankfurt.de

\section{D.R. Oliinychenko} \\ Bogoliubov Laboratory of Theoretical Physics, JINR, Joliot-Curie str. 6, Dubna, Russia \\ E-mail: Dimafopfegmail.com
}

\section{E.G. Nikonov}

Laboratory for Information Technologies, JINR, Joliot-Curie str. 6, 141980 Dubna, Russia

E-mail: E.Nikonovejinr.ru

\section{A.S. Sorin}

Bogoliubov Laboratory of Theoretical Physics, JINR, Joliot-Curie str. 6, Dubna, Russia

E-mail: Sorinetheor.jinr.ru

\section{G.M. Zinovjev}

Bogolyubov Institute for Theoretical Physics, Metrologichna str. $14^{B}$, Kiev 03680, Ukraine E-mail: Gennady.Zinovjev@cern.ch

Here we develop a model equation of state which successfully parameterizes the thermodynamic functions of hadron resonance gas model at chemical freeze-out and which allows us to naturally explain the adiabatic chemical freeze-out criterion. The present model enables us to clearly demonstrate that at chemical freeze-out the resulting hadronic mass spectrum used in the hadron resonance gas model is not an exponential-like, but a power-like. We argue that such a property of hadronic mass spectrum at chemical freeze-out can be explained by the two new effects found here for wide resonances existing in a thermal environment: the near threshold thermal resonance enhancement and the near threshold resonance sharpening. The effect of resonance sharpening is studied for a sigma meson and our analysis shows that for the temperatures well below 92 $\mathrm{MeV}$ the effective width of sigma meson is about 50 to $70 \mathrm{MeV}$. Thus, the effect of resonance sharpening justifies the usage of the sigma-like field-theoretical models for the strongly interacting matter equation of state at such temperatures. Also we argue that the most optimistic hope to find the quark gluon bags experimentally may be related to their sharpening and enhancement in a thermal medium. In this case the wide quark gluon bags may appear directly or in decays as narrow resonances that are absent in the tables of elementary particles and that have the width about $50-150 \mathrm{MeV}$ and the mass about or above $2.5 \mathrm{GeV}$.

XXI International Baldin Seminar on High Energy Physics Problems,

September 10-15, 2012

JINR, Dubna, Russia

${ }^{*}$ Speaker. 


\section{Introduction}

The hadron resonance gas model [1] is a reliable theoretical tool to extract information about the chemical freeze-out (FO) stage of the relativistic heavy ion collisions. However, the question about the reliable chemical FO criterion has a long history [1, 2, 3]. Very recently this question was thoroughly investigated again [4], using the most sophisticated version of the hadron resonance gas model. Similarly to [5] it was found that none of the formerly suggested chemical FO criteria [2] is robust, if the realistic particle table with the hadron masses up to $2.5 \mathrm{GeV}$ is used. However, in [4] the criterion of the adiabatic chemical FO was suggested. In [4] it was also shown that despite an essential difference with the model used in [1] the same conclusion on the constant entropy per particle at chemical FO is well reproduced by the chemical FO parameters found in $[1,5]$. Furthermore, such a criterion of chemical FO is also consistent with the best description of the Strangeness Horn puzzle found recently in [6]. Thus, it turns out that the criterion of the constant entropy per particle at chemical FO is, indeed, the reliable one. It is interesting that the constant entropy per particle at chemical FO was also found in [7], but the way of hard core repulsion used in this work is too different from the traditional one used in the hadron resonance gas model $[1,4,5]$ and, hence, in contrast to the results of [4,5], the model used in [7] leads to the simultaneous fulfillment of a few chemical FO criteria.

Despite the long history of this question until recently there was no a single attempt to understand what is the physical reason behind any of the chemical FO criterion. Only last year this problem got an adequate interest of theoreticians [3]. Here we develop an alternative approach and present a simple model equation of state which not only well describes the chemical FO thermodynamic parameters and reproduces the constant value of the entropy per particle at the chemical FO, but in addition it allows us to elucidate the real mass spectrum of mesons and baryons that generate such a criterion. As it will be shown below the real mass spectrum of hadrons, i.e. the density of hadronic states, is very much different from the Hagedorn mass spectrum which is traditionally expected to emerge already for hadrons with masses above $1.2 \mathrm{GeV}[8,9,10]$. However, below we demonstrate that the real mass spectrum of hadrons extracted from the adiabatic chemical FO model is not an exponential, but a power-like and the reason for such a behavior is an existence of many wide resonances. In this work we also show how at chemical FO a thermal medium essentially modifies the resonance mass distribution in case of large width leading to their narrowing near the threshold. Based on these findings we suggest that the quark-gluon bags may be observed at the NICA energy range as the narrow resonances (width about 50-150 MeV) with the mass about or above 2.5 which are absent in the tables of elementary particle properties.

The work is organized as follows. In Section 2 we present a model equation of state and extract its parameters from the data. The real mass spectrum of hadrons is extracted from the model and compared to the empirical one in Section 3. In Section 4 we analyze a mass attenuation of wide resonances in a thermal environment and discuss the related effects. Also in this section we argue on how the near threshold resonance enhancement affects the mass spectrum of hadrons at chemical FO. In Section 5 we perform a similar analysis for the quark-gluon bags are discuss the question how they can look like in the experiments. Our conclusions are contained in Section 6. 


\section{Model formulation}

It is, indeed, a remarkable fact that at the chemical FO the entropy per particle deviates from 7.18 by about $8 \%$ while the center of mass energy of collision changes from $\sqrt{s_{N N}}=2.2 \mathrm{GeV}$ to $\sqrt{s_{N N}}=7 \mathrm{TeV}$ [4]. In order to find the validity reason of the adiabatic chemical FO criterion we parameterize the mesonic (with subscript $M$ ) and baryonic (with subscript $B$ ) pressure as follows

$$
\begin{aligned}
& p_{M}=C_{M} T^{A_{M}} \exp \left[\frac{\mu_{M}-m_{M}}{T}\right], \\
& p_{B}=C_{B} T^{A_{B}} \exp \left[\frac{\mu_{B}-M_{B}}{T}\right],
\end{aligned}
$$

assuming that the integrated mass spectrum of mesons and baryons can be represented by the constants $C_{a}, A_{a}, m_{a}$, and $\mu_{M}$ with $a \in\{M, B\}$. Here $T$ denotes the chemical FO temperature and $\mu_{B}$ is the corresponding baryonic chemical potential. The pressure of antibaryons is, evidently, related to that one of baryons as $p_{\bar{B}}\left(T, \mu_{B}\right)=p_{B}\left(T,-\mu_{B}\right)$. Note that Eqs. (2.1) and (2.2) represent the mixture of gases of massive particles with the temperature dependent number of degrees of freedom without the hard-core repulsion. The thorough inspection $[1,4]$ shows that for the realistic values of hadronic hard-core radii below $0.45 \mathrm{fm}$ the effect of hard-core repulsion can be safely neglected for temperatures below 150-170 MeV. This fact is implemented in Eqs. (2.1) and (2.2).

Using the standard thermodynamic identities from Eqs. (2.1) and (2.2) one can find the particle densities $\rho_{a}=\frac{\partial p_{a}}{\partial \mu_{a}}=p_{a} / T$ with $a \in\{M, B, \bar{B}\}$ and entropy density $s \equiv \frac{\partial\left(p_{M}+p_{B}+p_{\bar{B}}\right)}{\partial T}$ at chemical FO and get the following expression for entropy per particle

$$
\frac{s}{\rho_{P}}=\frac{\left(A_{M}+\frac{m_{M}-\mu_{M}}{T}\right) \rho_{M}+\left(A_{B}+\frac{M_{B}}{T}\right)\left(\rho_{B}+\rho_{\bar{B}}\right)-\frac{\mu_{B}}{T}\left(\rho_{B}-\rho_{\bar{B}}\right)}{\rho_{M}+\rho_{B}+\rho_{\bar{B}}},
$$

where the particle density is denoted as $\rho_{P} \equiv \rho_{M}+\rho_{B}+\rho_{\bar{B}}$. In order to qualitatively explain the reason of constant ratio $s / \rho_{P}$ we note that there exist two regions where the baryonic chemical potential roughly linearly decreases with the FO temperature $[4,1]$, i.e. $\mu_{B}(T) \simeq \mu_{B}\left(T_{0}\right)+\mu_{B}^{\prime}(T-$ $\left.T_{0}\right) \simeq \mu_{B}\left(T_{0}\right)-\mu_{B}^{\prime} T_{0}+\mu_{B}^{\prime} T \simeq \mu_{B}^{0}+\mu_{B}^{\prime} T$. Taking the latter into account, one can write (2.3) as

$$
\frac{s}{\rho_{P}} \simeq \frac{\left(A_{M}+\frac{m_{M}-\mu_{M}}{T}\right) \rho_{M}+\left(A_{B}-\mu_{B}^{\prime}+\frac{M_{B}-\mu_{B}^{0}}{T}\right)\left(\rho_{B}+\rho_{\bar{B}}\right)+2 \frac{\mu_{B}}{T} \rho_{\bar{B}}}{\rho_{M}+\rho_{B}+\rho_{\bar{B}}} .
$$

Consider, first, the chemical FO temperatures below $135 \mathrm{MeV}[1,4]$. Note that in (2.4) the term $\frac{\mu_{B}}{T} \frac{\rho_{\bar{B}}}{\rho_{M}+\rho_{B}+\rho_{\bar{B}}} \ll 1$ since at large values of the baryonic chemical potentials the antibaryons are absent, while their number becomes comparable with the number of baryons for $\frac{\mu_{B}}{T} \rightarrow 0$. Now it is clear that one can get rid of the strong temperature dependence in (2.4), if the following conditions are obeyed

$$
\frac{m_{M}-\mu_{M}}{T} \simeq 0, \quad \frac{M_{B}-\mu_{B}^{0}}{T} \simeq 0 .
$$

Then the entropy per particle at chemical FO can be cast as

$$
\frac{s}{\rho_{P}} \simeq A_{B}-\mu_{B}^{\prime}-\left(A_{B}-\mu_{B}^{\prime}-A_{M}\right) \frac{\rho_{M}}{\rho_{M}+\rho_{B}+\rho_{\bar{B}}} \simeq A_{B}-\mu_{B}^{\prime},
$$


i.e. up to a small correction the entropy per particle is constant. Strictly speaking, the above rough estimates should be valid only for low chemical FO temperatures, when the number of mesons is, indeed, a correction to the number of baryons. However, at high chemical FO temperatures the baryonic chemical potential is also linearly dependent on $T[4,1]$, although with another value of the derivative $\tilde{\mu}_{B}^{\prime}$. Then at high chemical FO temperatures the entropy density per particle (2.5) becomes $\frac{s}{\rho_{P}} \simeq A_{B}-\tilde{\mu}_{B}^{\prime}-\left(A_{B}-\tilde{\mu}_{B}^{\prime}-A_{M}\right) \frac{\rho_{M}}{\rho_{M}+\rho_{B}+\rho_{\bar{B}}} \simeq A_{M}+\left(A_{B}-\tilde{\mu}_{B}^{\prime}-A_{M}\right) \frac{\left(\rho_{B}+\rho_{\bar{B}}\right)}{\rho_{M}+\rho_{B}+\rho_{\bar{B}}} \simeq$ Const, since at these temperatures the fraction of baryons and antibaryons is almost a constant. Thus, the entropy per particle at chemical FO is basically defined by the power $A_{a}$ of the dominated degrees of freedom ( $a=B$ for low T and $a=M$ for high T) and by the speed of baryonic chemical potential change with the temperature change, i.e. by $\mu_{B}^{\prime}$.

The above simplified consideration is well supported by a quantitative analysis. To demonstrate this in Fig. 1 we show the results of fit of the particle densities obtained in [4] for chemical FO. As one can see from Fig. 1 the quality of the chemical FO data for mesons (left panel) is much higher than that one of the baryons and antibaryons and, hence, the corresponding description of meson densities is excellent, while the description of baryon-antibaryon densities is good since the value of $\chi^{2} / d o f=7.8 / 11$ is acceptable. The major part of this $\chi^{2} / d o f$ value is generated by the three data points of the baryon-antibaryon density for the highest temperatures. We found the same minimum, if these three points are excluded from the fit, although in this case the corresponding value of $\chi^{2} /$ dof is much better $\chi^{2} / d o f=1.2 / 8$. Our analysis shows that such a defect of the present model can be repaired by the excluded volume corrections. However, here we prefer to keep the model as simple as possible in order to demonstrate the main idea and, hence, hereafter we do not consider the last three data points into further fitting.
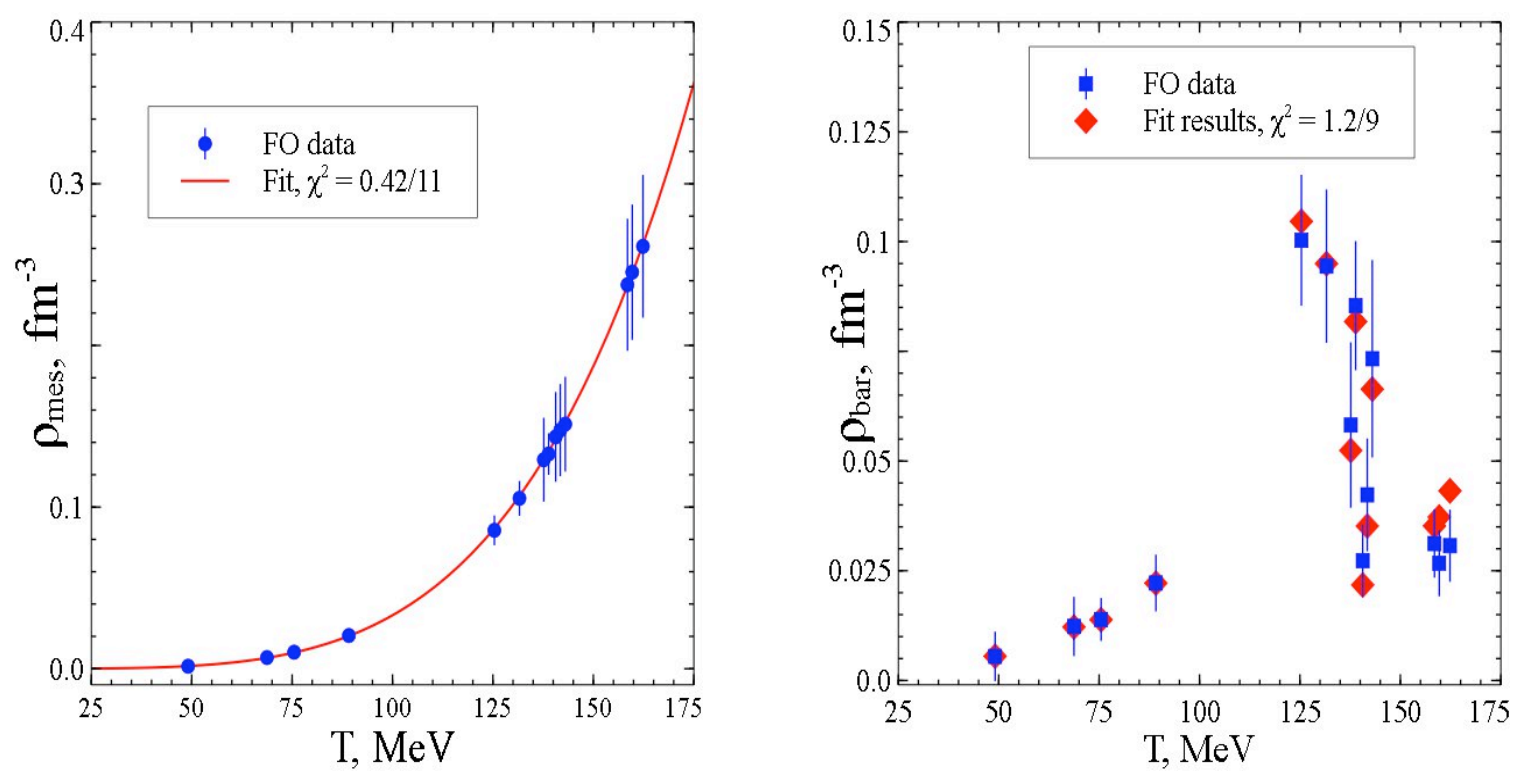

Figure 1: Temperature dependence of meson particle density $\rho_{M}$ (left panel) and baryon and antibaryon particle density $\left(\rho_{B}+\rho_{\bar{B}}\right)$ (right panel) for the developed model. The data points with error bars are the results of the fit obtained in [4] for the experimental hadronic multiplicities. 
The results of the density fit are as follows

$$
\begin{aligned}
& M_{B} \simeq 800.5 \pm 30 \mathrm{MeV}, \quad m_{M}-\mu_{M} \simeq-5 \pm 5 \mathrm{MeV}, \\
& C_{B} \simeq(2.624 \pm 0.191) \cdot 10^{-9} \frac{\mathrm{MeV}^{1-A_{B}}}{\mathrm{fm}^{3}}, \quad C_{M} \simeq(7.61 \pm 0.12) \cdot 10^{-9} \frac{\mathrm{MeV}^{1-A_{M}}}{\mathrm{fm}^{3}} \\
& A_{B} \simeq 6.097 \pm 0.38, \quad A_{M} \simeq 5.31 \pm 0.14 .
\end{aligned}
$$

Note that the conditions (2.5) are well reproduced by the fitting of the densities. It may, however, look strange that for the vanishing value of mesonic chemical potential the meson mass parameter $m_{M}$ in (2.7) can be negative. We note that the results of fitting show that condition (2.5) for mesons is numerically well established, since the minimal value of the chemical FO temperature is about $50 \mathrm{MeV}$. Therefore, the result (2.7) for mesons means that within the error bars one has $\mu_{M}=0$ and $m_{M}=0$.

Using the above parameters we calculated (no additional fit!) the entropy density and found its ratio to the particle density. The results are shown in the left panel of Fig. 2. From this figure one can see that only the three points at highest chemical FO temperatures which were not included into a fit are not well reproduced. Of course, as it is seen from the right panel of Fig. 2 the additional fitting of the ratio $s / \rho_{P}$ can improve the quality description of all analyzed points and one gets $\chi^{2} /$ dof $\simeq 0.47$.
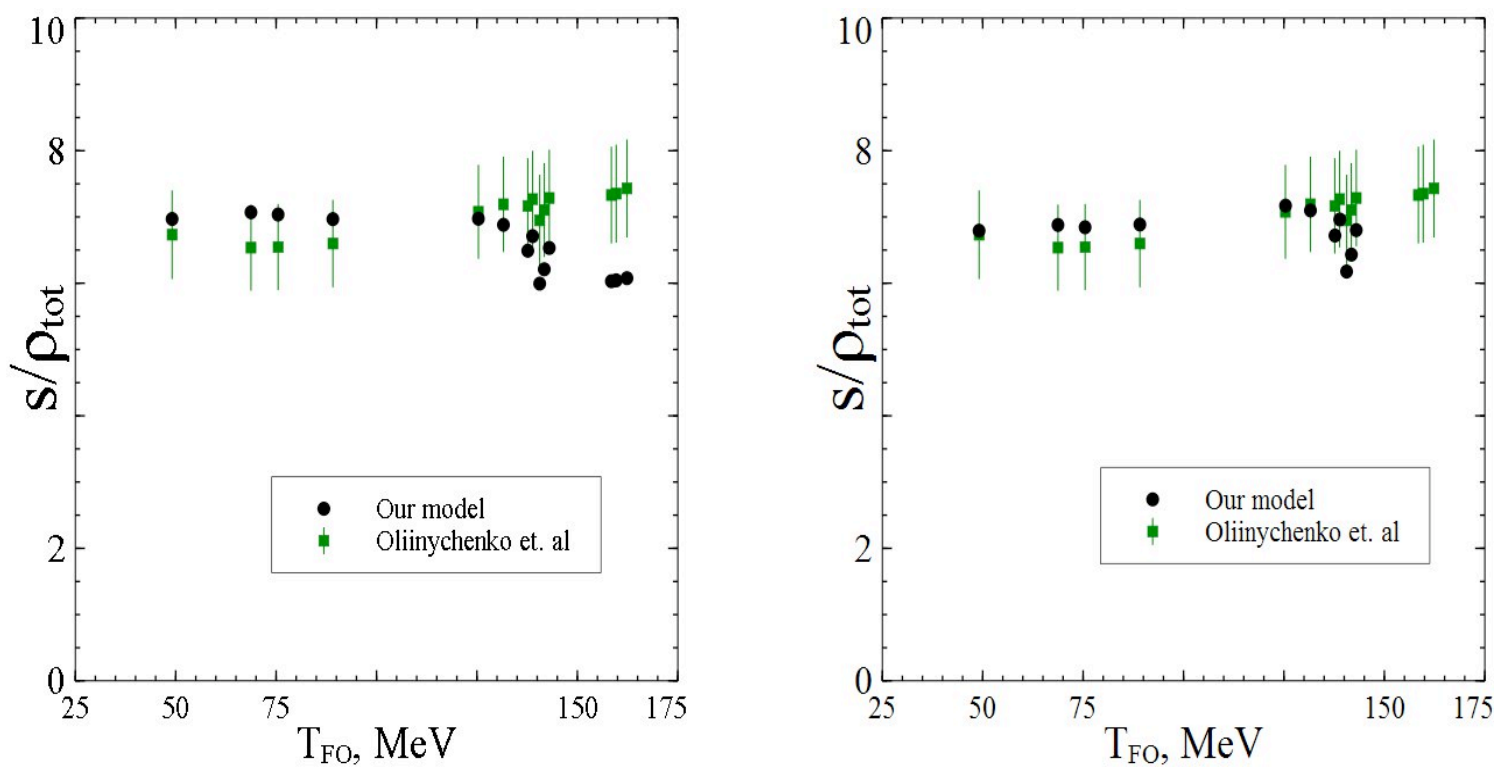

Figure 2: Left panel: Comparison of the chemical FO temperature dependence of the entropy per particle $s / \rho_{P}$ for the developed model (circles) and for the chemical FO data found in [4] (squares with error bars). Note that this ratio was just calculated using the parameters of Eqs. (2.7)-(2.9), i.e. no fitting was used. Right panel: the same ratio as in the left panel, but fitted together with particle densities.

\section{Extracted mass spectrum of hadrons}

The model equations (2.1) and (2.2) allow us to directly find the mass spectrum of hadrons. The question of whether the experimental mass spectrum of hadrons given in the Particle Data 
Group tables coincides with the spectrum suggested by R. Hagedorn is of great interest nowadays $[8,9,10]$. However, almost all discussions of the hadron mass spectrum simply ignore the width of resonances, whereas the large resonance width may essentially modify the spectrum $[11,12,13$, $14,15,16,17,18]$ and it may be even responsible for an absence of heavy excited resonances in the empirical mass spectrum of hadrons $[18,19]$. Therefore, it is interesting to study the effective mass spectrum of hadrons having the real width. Since in the hadron resonance gas model $[1,4]$ the resonance width is considered explicitly, its effect is also implicitly accounted for in the model equations (2.1) and (2.2).

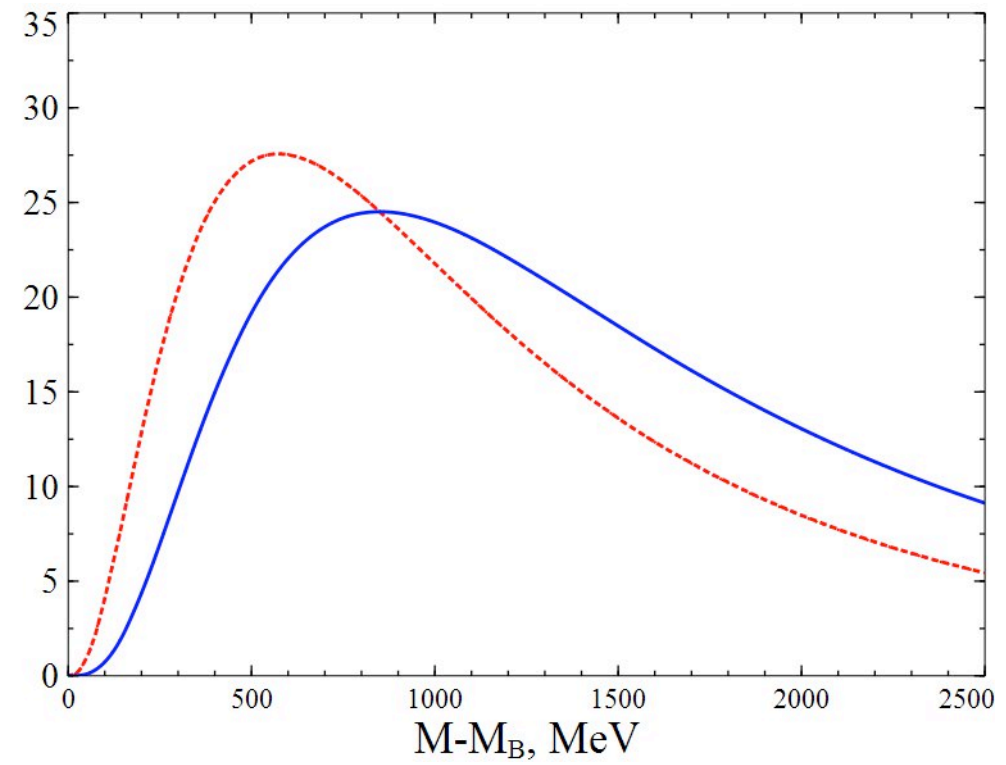

Figure 3: Ratio of the baryonic mass spectrum $\rho_{B}(m)$ of the present model found at chemical FO defined by (3.2) to the mass spectrum of all hadrons $\rho_{B}^{\exp }(m)$ taken from the Particle Data Group [20] and parameterized according to (3.3) [21]. The full curve shows the ratio of integrated spectra $\rho_{B}(m) / \rho_{B}^{\exp }(m)$, while the dotted curve demonstrates the ratio of the corresponding densities of states. The mass $M_{B}=800.5 \mathrm{MeV}$ is taken from Eq. (2.7).

In order to elucidate the effective mass spectrum of baryons we rewrite their density in terms of the mass integral of the momentum integral of the Boltzmann distribution function. Since the effective baryon mass $M_{B}$ in (2.7) is large compared to the maximal value of the chemical FO temperature, one can safely use the non-relativistic approximation for the momentum integration. This means that the factor $T^{\frac{3}{2}}$ should be assigned to the momentum integration, while the remaining T-dependence of the baryon (and antibaryon) particle density can be identically cast as

$$
\begin{aligned}
\rho_{B} & =C_{B} T^{A_{B}-1} \exp \left[\frac{\mu_{B}-M_{B}}{T}\right]=C_{B} \exp \left[\frac{\mu_{B}}{T}\right] \int_{M_{B}}^{\infty} d m \frac{\left(m-M_{B}\right)^{A_{B}-3.5}}{\Gamma\left(A_{B}-2.5\right)} T^{\frac{3}{2}} \exp \left[-\frac{m}{T}\right] \\
& \simeq(2 \pi)^{\frac{3}{2}} C_{B} \exp \left[\frac{\mu_{B}}{T}\right] \int_{M_{B}}^{\infty} d m \frac{\left(m-M_{B}\right)^{A_{B}-3.5}}{m^{\frac{3}{2}} \Gamma\left(A_{B}-2.5\right)} \int \frac{d^{3} k}{(2 \pi)^{3}} \exp \left[-\frac{\sqrt{k^{2}+m^{2}}}{T}\right],
\end{aligned}
$$

where in the last step of derivation we accounted for the fact that besides $\exp \left[-\frac{m}{T}\right]$ the momentum 
integration in non-relativistic case generates the factor $(2 \pi m T)^{\frac{3}{2}}$. Here $\Gamma(A)$ is the usual gamma function and, hence, one has $\Gamma(3.6) \simeq 3.717$.

Since the original mass integral in (3.1) is the Laplace integral, then the representation (3.1) uniquely defines the $T$-dependence of baryonic density and vice versa. Thus, from (3.1) one concludes that at chemical FO the effective mass spectrum of baryons and antibaryons is rather a power-like than an exponential-like:

$$
\frac{\partial \rho_{B}(m)}{\partial m} \simeq \frac{(2 \pi)^{\frac{3}{2}} \hbar^{3} C_{B}}{\Gamma(3.6)} \frac{\left(m-M_{B}\right)^{2.6}}{m^{\frac{3}{2}}},\left.\quad \Rightarrow \quad \rho_{B}(m)\right|_{m \gg M_{B}} \sim m^{2.1} .
$$

Comparing the baryonic mass spectrum $\rho_{B}(m) \equiv \int_{M_{B}}^{m} d z \frac{\partial \rho_{B}(z)}{\partial z}$ obtained from the density of states (3.2) with the parameterization

$$
\rho_{B}^{\exp }(m) \simeq\left[\frac{m}{537 \mathrm{MeV}}\right]^{5.72},
$$

suggested in [21] to describe the experimental hadronic mass spectrum, one finds a great disagreement between them! Indeed, as one can see from Fig. 3 these two mass spectra may deviate from each other by about 25 times at $m \simeq 1650 \mathrm{MeV}$ and their asymptotic behaviors are completely different, since $\rho_{B}(m) \simeq m^{2.1}$ in (3.2), while $\rho_{B}^{e x p}(m) \simeq m^{5.72}$ in (3.3). Naturally, there arise two questions, "Does it mean that one of these two mass spectra is wrong?" and "What is the reason for so huge difference between these mass spectra?"

\section{Effective width of wide resonances}

In order to demonstrate that none of these two mass spectra is wrong and that at chemical FO the large difference between these two hadronic mass spectra is due to the width of hadronic resonances, we consider the Gaussian mass attenuation instead of the Breit-Wigner one that is used in the actual simulations since in this case the evaluation is more transparent. Also such a treatment would allow us to obtain some important conclusions on the mass spectrum of quark-gluon (QG) bags which according to $[18,19]$ should unavoidably have the Gaussian mass attenuation. Note also that the estimates below provide us with the lower limit, since the Gaussian mass distribution vanishes much faster than the Breit-Wigner one. The typical term of the $k$-resonance that enters into the mass spectrum of the baryonic particle density of the hadron resonance gas model is given by $F_{k}\left(\sigma_{k}\right) \exp \left[\frac{\mu_{B}}{T}\right][1,4]$, where

$$
F_{k}\left(\sigma_{k}\right) \equiv g_{k} \int_{0}^{\infty} d m \frac{\Theta\left(m-M_{k}^{T h}\right)}{N_{k}\left(M_{k}^{T h}\right)} \exp \left[-\frac{\left(m_{k}-m\right)^{2}}{2 \sigma_{k}^{2}}\right] \int \frac{d^{3} p}{(2 \pi \hbar)^{3}} \exp \left[-\frac{\sqrt{p^{2}+m^{2}}}{T}\right] .
$$

Here $m_{k}$ is the mean mass of the $k$-th resonance, $g_{k}$ is its degeneracy factor, $\sigma_{k}$ is the Gaussian width which defines the true width of such a resonance as $\Gamma_{k}=Q \sigma_{k}$ (with $Q \equiv 2 \sqrt{2 \ln 2}$ ) and the normalization factor is defined via the threshold mass $M_{k}^{T h}$ of the dominant channel as $N_{k}\left(M_{k}^{T h}\right) \equiv$ $\int_{M_{k}^{T h}}^{\infty} d m \exp \left[-\frac{\left(m_{k}-m\right)^{2}}{2 \sigma_{k}^{2}}\right]$. For the narrow resonances the term $F_{k}\left(\sigma_{k}\right)$ converts into the usual thermal 

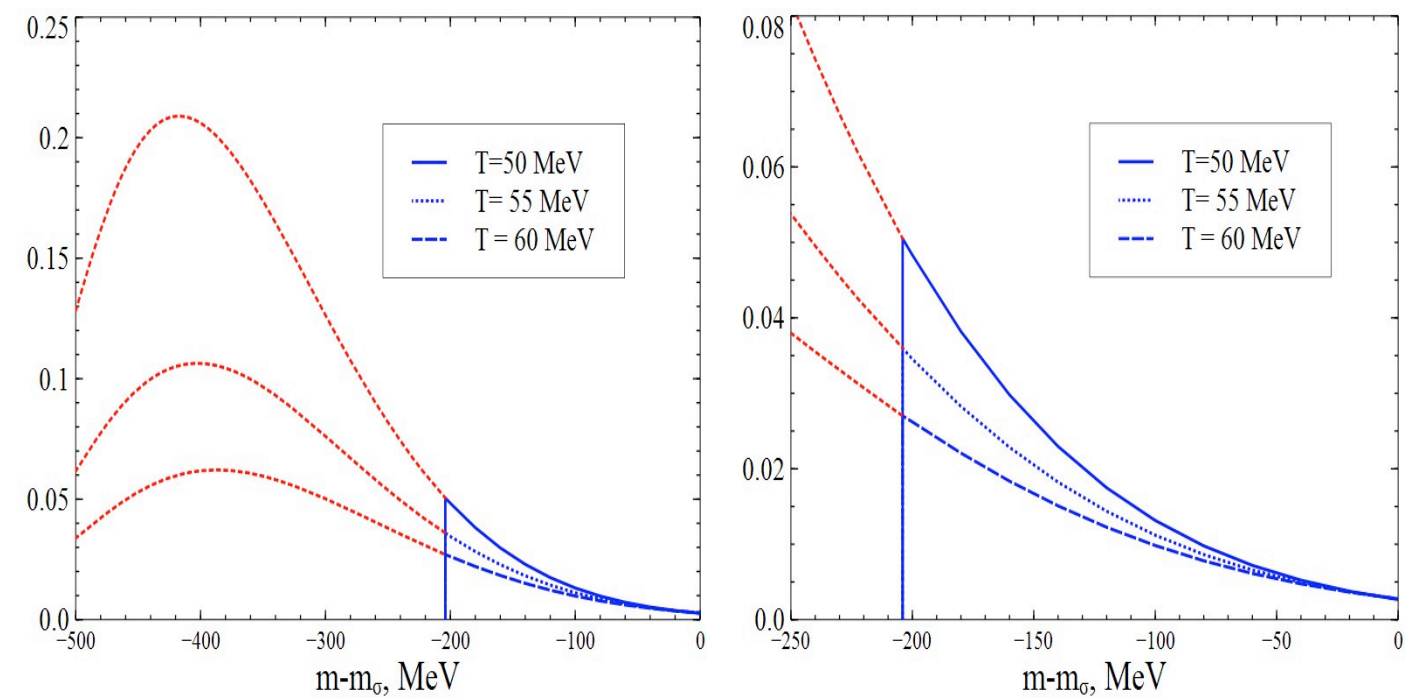

Figure 4: Temperature dependence of the mass distribution $f_{k}(m) / \phi\left(m_{\sigma}, T\right.$ ) (in units of $1 / \mathrm{MeV}$, see Eq. (4.2)) for $\sigma$-meson with the mass $m_{\sigma}=484 \mathrm{MeV}$, the width $\Gamma_{\sigma}=510 \mathrm{MeV}[25]$ and $M_{\sigma}^{T h}=2 m_{\pi} \simeq 280 \mathrm{MeV}$. In the left panel the short dashed curves below the two pion threshold (vertical line at $m-m_{\sigma}=-204 \mathrm{MeV}$ ) show the mass attenuation which does not contribute into the particle density (4.2). From the right panel one can see the effect of wide resonance sharpening near the threshold, i.e. an appearance of a narrow peak in the resulting mass distribution on the right hand side of the threshold which resembles an icy slide. For different temperatures this mass attenuation is shown by the solid, the short dashed and the long dashed curves. The $\sigma$-meson effective width was found numerically from these mass attenuations: $\Gamma_{\sigma}^{e f f}(T=50 \mathrm{MeV}) \simeq 62.5$ $\mathrm{MeV}, \Gamma_{\sigma}^{e f f}(T=55 \mathrm{MeV}) \simeq 71.5 \mathrm{MeV}$ and $\Gamma_{\sigma}^{e f f}(T=60 \mathrm{MeV}) \simeq 82.5 \mathrm{MeV}$

density of particles, i.e. for $\sigma_{k} \rightarrow 0$ one has $F_{k} \rightarrow g_{k} \phi\left(m_{k}, T\right)$, where the following notation is used $\phi(m, T) \equiv \int \frac{d^{3} p}{(2 \pi \hbar)^{3}} \exp \left[-\frac{\sqrt{p^{2}+m^{2}}}{T}\right]$.

A usage of Eq. (4.1) was heavily criticized in [24], but we find such a critique absolutely inadequate for the states below the chemical FO. First of all, we note that in the approach of [24] the effect of medium cannot be switched off at any finite particle density or temperature. This means that according to the treatment of [24] (and many similar works!) all the hadrons whose momentum spectra are frozen due to the absence of any strong interaction between them should keep their momentum dependent width and mass which they acquired at the moment of kinetic FO up to they are captured by the detectors. Hence, according to [24] all hadrons measured by detectors, including the stable ones, are some resonances that 'feel' a thermal medium in which they were produced long after the medium is gone. Second, all the 'effects' which the authors of [24] claim to be of principal physical importance are reduced to a slight (by about $20 \mathrm{MeV}$ ) shift of the mass attenuation peak and small change of its shape for the $\Delta_{33}$ resonance, although the corresponding modification of the nucleon and pion properties the authors of [24] simply ignore. In our opinion such modifications of the mass attenuation of the $\Delta_{33}$ resonance compared to the 'crude' approximation of Eq. (4.1) cannot be measured in heavy ion experiments even for such narrow resonances as $\Delta_{33}$. Therefore, a serious discussion of similar 'effects' for heavy hadronic resonances whose mass and width are often known with the accuracy of $100 \mathrm{MeV}$ or $200 \mathrm{MeV}$ (or worse) [20] does not make any sense. Thus, at the present state of art there is no alternative to a 
physically transparent Eq. (4.1) to be used at and after the moment of chemical FO.
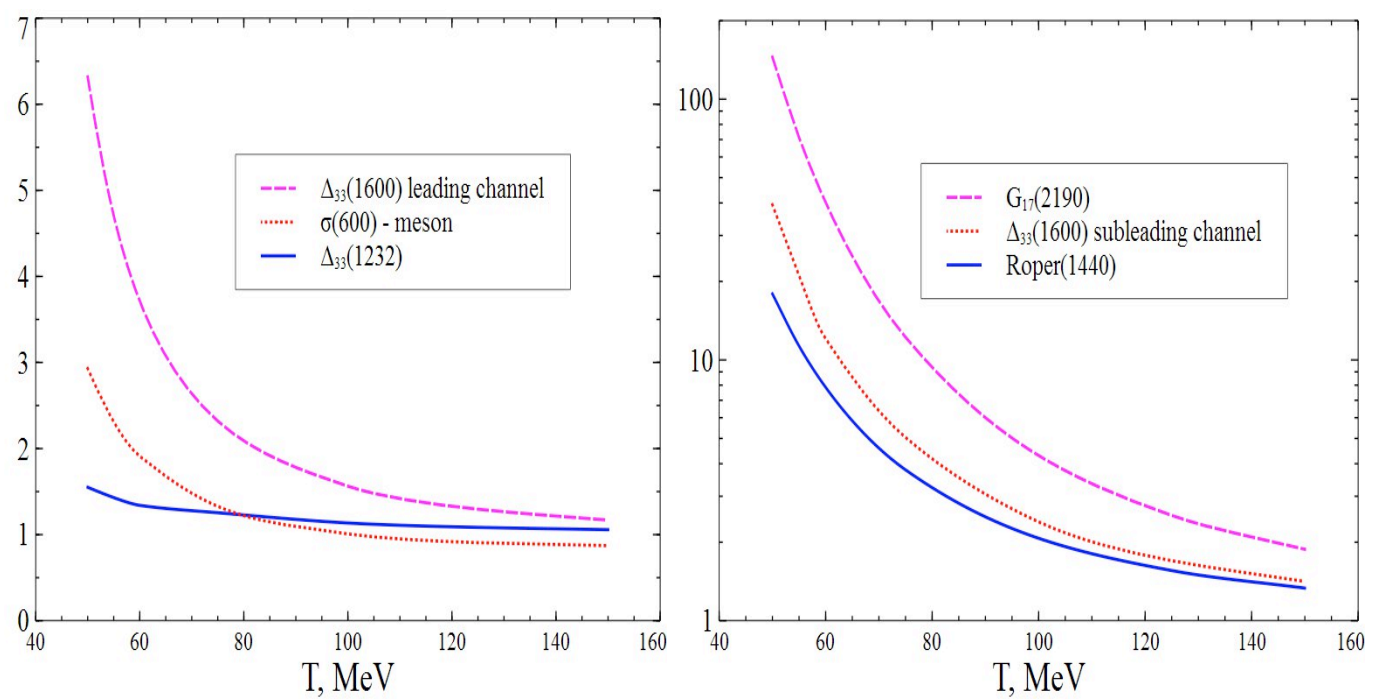

Figure 5: Temperature dependence of the resonance enhancement. The ratio $R(T)=\frac{f_{k}}{\phi\left(m_{k}, T\right)}$ is shown for the hadronic resonance decays given in Table I. For wide resonances the effect of enhancement can be huge.

The momentum integral in (4.1) can be written using the non-relativistic approximation $\phi(m, T) \simeq$ $\left[\frac{m T}{2 \pi \hbar^{2}}\right]^{\frac{3}{2}} \exp \left[-\frac{m}{T}\right]$. Then to simplify the mass integration of (4.1) one can make the full square in it from the powers of $\left(m_{k}-m\right)$ and get

$$
F_{k}\left(\sigma_{k}\right) \equiv g_{k} \int_{0}^{\infty} d m f_{k}(m) \simeq \tilde{g}_{k} \int_{0}^{\infty} d m \frac{\Theta\left(m-M_{k}^{T h}\right)}{N_{k}\left(M_{k}^{T h}\right)} \exp \left[-\frac{\left(\tilde{m}_{k}-m\right)^{2}}{2 \sigma_{k}^{2}}\right]\left[\frac{m T}{2 \pi \hbar^{2}}\right]^{\frac{3}{2}} \exp \left[-\frac{m_{k}}{T}\right],(
$$

where the following notations for an effective resonance degeneracy $\tilde{g}_{k}$ and for an effective resonance mass $\tilde{m}_{k}$

$$
\begin{aligned}
& \tilde{g}_{k} \equiv g_{k} \exp \left[\frac{\sigma_{k}^{2}}{2 T^{2}}\right]=g_{k} \exp \left[\frac{\Gamma_{k}^{2}}{2 Q^{2} T^{2}}\right], \\
& \tilde{m}_{k} \equiv m_{k}-\frac{\sigma_{k}^{2}}{T}=m_{k}-\frac{\Gamma_{k}^{2}}{Q^{2} T},
\end{aligned}
$$

are used. From Eq. (4.2) one can see that the presence of the width, firstly, may strongly modify the degeneracy factor $g_{k}$ and, secondly, it may essentially shift the maximum of the mass attenuation towards the threshold or even below it. There are two corresponding effects which we named as the near threshold thermal resonance enhancement and the near threshold resonance sharpening. These effects formally appear due to the same reason as the famous Gamow window for the thermonuclear reactions in stars [22, 23]: just above the resonance decay threshold the integrand in (4.2) is a product of two functions of a virtual resonance mass $m$, namely, the Gaussian attenuation is an increasing function of $m$, while the Boltzmann exponent strongly decreases above the threshold. The resulting attenuation of their product has a maximum, whose shape, in contrast to the usual Gamow window, may be extremely asymmetric due to the presence of the threshold. Indeed, as one can see from Fig. 4 the resulting mass attenuation of a resonance may acquire the form 
of the sharp and narrow peak that is closely resembling an icy slide. Below we discuss these two effects is some details. Qualitatively the same effects appear, if in (4.2) one uses the Breit-Wigner resonance mass attenuation instead of the Gaussian one.

\begin{tabular}{|c|c|c|c|c|c|c|c|c|}
\hline Hadron & $\begin{array}{c}m_{k} \\
(\mathrm{MeV})\end{array}$ & $\begin{array}{c}\Gamma_{k} \\
(\mathrm{MeV})\end{array}$ & $\begin{array}{c}\text { Decay } \\
\text { channel }\end{array}$ & $\begin{array}{c}M_{k}^{T h} \\
(\mathrm{MeV})\end{array}$ & $\beta_{k}$ & $\begin{array}{c}T_{k}^{+} \\
(\mathrm{MeV})\end{array}$ & $\begin{array}{c}\Gamma_{k}^{\text {eff }} \\
(\mathrm{MeV})\end{array}$ & $\begin{array}{c}\Gamma_{k}^{N} \\
(\mathrm{MeV})\end{array}$ \\
\hline$\sigma$-meson & 484 & 510 & $\sigma \rightarrow \pi \pi$ & 280 & 0.942 & 91.9 & 62.5 & 67.3 \\
\hline$P_{33}$ & 1232 & 120 & $\Delta \rightarrow \pi N$ & 1080 & 2.98 & 11.6 & 43.5 & N/A \\
\hline$P_{11}$ & 1440 & 350 & $N \rightarrow \pi N$ & 1080 & 2.42 & 38.74 & 129.5 & N/A \\
\hline$P_{33}$ & 1600 & 350 & $\Delta \rightarrow \pi \Delta$ & 1372 & 1.53 & 50.4 & 68.7 & 80.8 \\
\hline$P_{33}$ & 1600 & 350 & $\Delta \rightarrow \pi N$ & 1080 & 3.5 & 30.3 & 280. & N/A \\
\hline$G_{17}$ & 2190 & 500 & $\Delta \rightarrow \rho N$ & 1710 & 2.26 & 57.8 & 74.6 & 81.8 \\
\hline
\end{tabular}

Table 1: The parameters of several hadronic resonances together with their decay channels that are used to determine the quantities $\beta_{k}$ and $T_{k}^{+}$. The last two columns show the corresponding effective width at temperature $T=50 \mathrm{MeV}$ found, respectively, numerically from Eq. (4.1) and analytically from Eq. (4.5), when it can be applied.

From the definitions of the effective resonance mass (4.4) and the effective resonance degeneracy (4.3) one can see that the effects of their change are strong for $T \ll \sigma_{k}$. This can be clearly seen from Fig. 4, which demonstrates both of the above effects at low temperatures for the $\sigma$-meson. A simple analysis shows that the effect of resonance sharpening is strongest, if the threshold mass is shifted to the convex part of the Gaussian distribution in (4.2), i.e. for $M_{k}^{T h} \geq \tilde{m}_{k}$ or for the temperatures $T$ below $T_{k}^{+} \equiv \frac{\sigma_{k}^{2}}{m_{k}-M_{k}^{T h}} \equiv \frac{\sigma_{k}}{\beta_{k}}$. To demonstrate the effect of the width sharpening we list a few typical examples for baryons in the Table 1. For $T<T_{k}^{+}$and for $m>M_{k}^{T h}$ the Gaussian mass distribution in (4.2) can be safely approximated as $\exp \left[-\frac{\left(\tilde{m}_{k}-m\right)^{2}}{2 \sigma_{k}^{2}}\right] \approx$ $\exp \left[-\frac{\left(\tilde{m}_{k}-M_{k}^{T h}\right)^{2}}{2 \sigma_{k}^{2}}-\frac{\left(\tilde{m}_{k}-M_{k}^{T h}\right)}{\sigma_{k}^{2}}\left(m-M_{k}^{T h}\right)\right]$. Now recalling the standard definition of the width for the function $f(x)=\Theta(x)$ Const $\exp [-b x]$, one obtains the temperature dependent resonance effective width near the threshold as

$$
\Gamma_{k}^{N}(T) \simeq \frac{\ln (2)}{\frac{1}{T}-\frac{\beta_{k}}{\sigma_{k}}} \equiv \frac{\ln (2)}{\frac{1}{T}-\frac{1}{T_{k}^{+}}},
$$

since for such a distribution function $f(x)$ one gets $f(\ln (2) / b)=f(0) / 2$. Note that in evaluating (4.5) we neglected the additional $m^{1.5}$-dependence in (4.2), but one can readily check that numerically such a correction is small. The rightmost column in Table 1 demonstrates that Eq. (4.5), indeed, provides an accurate estimate for $T<T_{k}^{+}$. The results of Table 1 also justify the usage of $\sigma$-meson and the field-theoretical models based on the well known $\sigma$-model for temperatures well below $T_{\sigma}^{+} \simeq 92 \mathrm{MeV}$. Of course, the present approach which is developed for the chemical FO stage, when the inelastic reactions except for resonance decays are ceased to exist, cannot be applied for earlier stages of heavy ion collisions. However, here we would like to stress that an inclusion of the large width of $\sigma$-meson in the field-theoretical models of the strongly interacting matter equation of state is very necessary. From the above analysis one can see that the large width 
inclusion can generate some new important physical effects like the wide resonance sharpening in a thermal medium.

From Fig. 5 one can see that the resonance enhancement can be, indeed, huge for wide $(\Gamma \geq$ $450 \mathrm{MeV})$ and medium wide $(\Gamma \simeq 300-400 \mathrm{MeV})$ resonances. This effect naturally explains the strong temperature dependence of hadronic pressure (2.1) at chemical FO, which in its turn generates the power-like mass spectrum of baryons (3.2). Clearly, the same is true for the mesonic pressure (2.2) and mesonic mass spectrum in a thermal environment. However, we believe that a detailed study of such a phenomenon requires a special investigation.

\section{Effective width of QG bags}

The first important conclusion from the analysis above is that there is no sense to discuss the mass spectrum of hadronic resonances, empirical or Hagedorn, without a treatment of their width. Furthermore, the same is true for the QG bags which, according to the finite width model $[18,19]$, are heavy and wide resonances with mass $M_{B}$ larger than $M_{0} \simeq 2.5 \mathrm{GeV}$ and with the mean width of the form $\Gamma_{B} \simeq \Gamma_{0}(T)\left[\frac{M_{B}}{M_{0}}\right]^{\frac{1}{2}}$, where $\Gamma_{0}(T)$ is a monotonically increasing function of $T$ and $\Gamma_{0}(T=$ $0) \in[400 ; 600] \mathrm{MeV}$. This range of $\Gamma_{0}(T=0)$ values corresponds to the cross-over temperatures $T_{c o} \simeq 170-200 \mathrm{MeV}[18,19]$ for vanishing baryonic density. The value $\Gamma_{0}(T=0)=400 \mathrm{MeV}$ is well consistent with the results of the present days lattice QCD thermodynamics [26, 27], but there is no guaranty that the lattice QCD data will not change in the future. Therefore, below we consider the whole range of values for the width $\Gamma_{0}(T)$ analyzed in $[18,19]$.

There are two interesting features of QG bags which are related to the above treatment. Thus, from the results of $[18,19]$ and from (4.5) one can find the temperature $T_{B}^{+}$for the QG bags as

$$
T_{B}^{+} \simeq \frac{\Gamma_{0}^{2}(T)}{Q^{2} M_{0}\left(1-\xi_{B}\right)} \simeq \frac{1}{\left(1-\xi_{B}\right)} \cdot \begin{cases}11.5-26 . \mathrm{MeV}, & \text { if } \Gamma_{0} \simeq 0.4-0.6 \mathrm{GeV} \text { at } T=0 \\ 46-104 \mathrm{MeV}, & \text { if } \Gamma_{0} \simeq 0.8-1.2 \mathrm{GeV} \text { at } T=90 \\ 140-315 \mathrm{MeV}, & \text { if } \Gamma_{0} \simeq 1.4-2.1 \mathrm{GeV} \text { at } T=170\end{cases}
$$

where $\xi_{B} \equiv \frac{M_{B}^{T h}}{M_{B}}$ denotes the ratio of the leading threshold mass $M_{B}^{T h}$ of the bag to its mean mass $M_{B}$. In (5.1) the temperature $T$ is given in $\mathrm{MeV}$. Clearly for different bags the range of $\xi_{B}$ value can be between 0 and 1 . Therefore, according to above results the bags with $\xi_{B} \rightarrow 1$ should have been essentially enhanced and sharpened as the ordinary resonances. Moreover, according to (4.5) in this case for $T \ll T_{B}^{+}$the QG bags should have had a small width $\Gamma_{B}^{N} \simeq \frac{T T_{B}^{+}}{T_{B}^{+}-T} \ln (2)$ and, hence, such QG bags should have been stable or, in other words, these bags should have been observed! The reason why such bags are not observed in the experiments is naturally explained by the finite width model $[18,19]$ : it is due to the effect called as the subthreshold suppression, i.e. a huge suppression (of about fifteen to sixteen orders of magnitude compared to light hadrons!) of the QG bags for temperatures below the half of the traditional Hagedorn temperature $T_{H}$ (for more details see a discussion after Eq. (42) in [19]). Such a suppression is a manifestation of the color confinement in terms of the QG bag width [19].

On the other hand Eq. (5.1) also shows that the only hope to observe the QG bags exists, if $\xi_{B} \rightarrow 1$. Then for chemical FO temperatures much below $T_{B}^{+}$such bags could have sufficiently long eigen lifetime of about $\tau_{B} \sim \frac{1}{\Gamma_{B}^{N}} \simeq \frac{T_{B}^{+}-T}{T T_{B}^{+} \ln (2)} \leq \frac{1}{T \ln (2)}$. Substituting $T \simeq 0.5 T_{H} \in[80 ; 90] \mathrm{MeV}$ in the 
last inequality and using the estimate of Eq. (5.1) for $T=90 \mathrm{MeV}$ with $\xi_{B}=0.9$, one finds the most optimistic estimate for the QG bag eigen lifetime as $\tau_{B} \leq 3.3 \pm 0.3 \mathrm{fm} / \mathrm{c}$. These estimates allow us to make the second important conclusion that an appearance of sharp resonances (baryonic or/and mesonic) with the width in the interval between 50 to $70 \mathrm{MeV}$ at the chemical FO temperatures close to $T_{Q G B} \simeq 85 \pm 5 \mathrm{MeV}$ that have the mass above $2.5 \mathrm{GeV}$ and that are absent in the tables of particle properties would be a clear signal of the $Q G$ bag formation. Their possible appearance at chemical FO as metastable states of finite systems created in relativistic nuclear collisions is justified by the finite width model [18, 19]. At higher temperatures such QG bags can be formed too, but their width is larger and lifetime is shorter. The limiting values of $\xi_{B}$ at $T$ for which the effect of resonance sharpening can exist is determined by the relation $\Gamma_{0}^{2}(T) / T \geq Q^{2} M_{0}\left(1-\xi_{B}\right)$ from which one can see that the condition $\xi_{B} \rightarrow 0.9$ can be relaxed, but in this case the temperature of chemical FO gets higher.

In addition one has to account for the statistical probability of the QG bags appearance at a given temperature $T$. Relatively to the nucleon the statistical probability of the QG bag of mass $M_{B}$ is about $W=\left[\frac{M_{B}}{M_{N}}\right]^{1.5} \exp \left[\frac{\left(M_{N}-M_{B}\right)}{T}\right] R_{B}(T)$, where $M_{N} \simeq 940 \mathrm{MeV}$ is the nucleon mass and $R_{B}(T)$ is the resonance enhancement factor in a thermal medium. For $T=140 \mathrm{MeV}$ and $M_{B}=M_{0} \simeq 2.5$ $\mathrm{GeV}$ one gets $W_{B} \simeq 3.85 \cdot 10^{-5} R_{B}$. Our analysis shows that for such temperatures the typical resonance effective width values are about $\Gamma_{B} \simeq 100-150 \mathrm{MeV}$ while the typical values of the resonance enhancement factor is about $R_{B} \simeq 10-100$ and, hence, compared to nucleon the relative statistical probability of such QG bags is about $W_{B} \simeq 3.85 \cdot\left(10^{-4}-10^{-3}\right)$, which is essentially larger than the relative probability of the $J / \psi$ meson $W_{J / \psi} \simeq 1.19 \cdot 10^{-6}$ at the same temperature. Note that the chemical FO temperature $T \simeq 140 \mathrm{MeV}$ corresponds to the highest SPS energy of collision at which the $J / \psi$ mesons are safely measured. Note that the chemical FO temperatures about $T_{Q G B} \simeq 80-140 \mathrm{MeV}$ correspond to the center of mass energy of collision $\sqrt{s_{N N}} \in[4 ; 8]$ $\mathrm{GeV}[1,4]$, which is in the range of the Dubna Nuclotron and NICA energies of collision. This energy range sets the most promising kinematic limit for the QG bag searches.

\section{Conclusions}

Here we present a simple model equation of state which successfully parameterizes the thermodynamic functions of hadron resonance gas model at chemical FO. Such a model with the temperature dependent number of hadronic degrees of freedom allowed us to naturally explain the adiabatic chemical freeze-out criterion that was found previously. In addition, this model allowed us to find out that the effective mass spectrum of baryons used in the resonance hadron gas model is not an exponential-like, but a power-like. Evidently, the same conclusion is valid for mesons, but such an analysis will be published elsewhere.

In order to give a reason for the obtained difference between the effective baryonic mass spectrum and the empirical hadronic mass spectrum we analyzed the behavior of wide resonances in a thermal environment and found two new effects occurring, if the chemical FO temperature is small compared to the resonance width: the near threshold thermal resonance enhancement and the near threshold resonance sharpening. Further analysis showed that for the temperatures well below 92 $\mathrm{MeV}$ the $\sigma$-meson can be rather narrow and it has the effective width of about 50 to $70 \mathrm{MeV}$. Thus, accounting for the $\sigma$-meson large width in a thermal medium allows us to justify the usage of the 
$\sigma$-like field-theoretical models for the strongly interacting matter equation of state for temperatures well below $92 \mathrm{MeV}$. Finally, we argued that the most optimistic hope to find the QG bags experimentally would be related to their sharpening and enhancement by a thermal medium. Then the QG bags may appear directly or in decays as narrow resonances of the width about $50-150 \mathrm{MeV}$ that have the mass about or above $2.5 \mathrm{GeV}$ and that are absent in the tables of elementary particles.

\section{Acknowledgments}

The authors appreciate the valuable comments of D. B. Blaschke, M. Bleicher and S. N. Nedelko. K.A.B., D.R.O. and G.M.Z. acknowledge the partial support of the Program 'On Perspective Fundamental Research in High Energy and Nuclear Physics' launched by the Section of Nuclear Physics and Energetics of National Academy of Sciences of Ukraine. The work of E.G.N. and A.S.S. was supported in part by the Russian Foundation for Basic Research, Grant No. 11-0201538-a.

\section{References}

[1] A. Andronic, P. Braun-Munzinger and J. Stachel, Nucl. Phys. A 772 (2006) 167.

[2] J. Cleymans, K. Redlich, Phys. Rev. Lett. 81 (1998) 5284.

[3] D.B. Blaschke, J. Berdermann, J. Cleymans and K. Redlich, Phys. Part. Nucl. Lett. 8 (2011) 811; Few Body Syst. 53 (2012) 99.

[4] D.R. Oliinychenko, K.A. Bugaev and A.S. Sorin, hep-ph/1204.0103.

[5] A. Andronic, P. Braun-Munzinger, J. Stachel and M. Winn, nucl-th/1201.0693.

[6] K. A. Bugaev, D. R. Oliinychenko, A. S. Sorin and G .M. Zinovjev, hep-ph/1208. 5968.

[7] S. K. Tiwari, P. K. Srivastava, and C. P. Singh, Phys. Rev. C 85 (2012) 014908.

[8] W. Broniowski, W. Florkowski and L. Y. Glozman, Phys. Rev. D 70 (2004) 117503.

[9] A. Andronic, P. Braun-Munzinger and J. Stachel, Acta Phys. Polon. B 40 (2009) 1005.

[10] J. Cleymans and D. Worku, Mod. Phys. Lett. A 26 (2011) 1197.

[11] D. Hahn and H. Stöcker, Nucl. Phys. A 452 (1986) 723.

[12] K. G. Denisenko and St. Mrowczynski, Phys. Rev. C 35 (1987) 1932.

[13] M.I. Gorenstein, St. Mrowczynski and D.H. Rischke, Phys. Lett. B 243 (1990) 327.

[14] J. Sollfrank, P. Koch and U. Heinz, Z. Phys. C 52 (1991) 593.

[15] K. A. Bugaev, M. I. Gorenstein and D. H. Rischke, Phys. Lett. B255 (1991) 18.

[16] D.B. Blaschke and K.A. Bugaev, Fizika B 13 (2004) 491.

[17] D.B. Blaschke and K.A. Bugaev, Prog. Part. Nucl. Phys. 53 (2004)197.

[18] K.A. Bugaev, V.K. Petrov and G.M. Zinovjev, Europhys. Lett. 85 (2009) 22002.

[19] K.A. Bugaev, V.K. Petrov and G.M. Zinovjev, Phys. Rev. C 79 (2009) 054913.

[20] C. Amsler et al., Phys. Lett. B 667 (2008) 1 [http://pdg.lbl.gov]. 
[21] T. D. Cohen and V. Krejcirik, hep-ph/1107.2130.

[22] C. S. Rolfs and W. S. Rodney, Cauldrons in the Cosmos, University of Chicago Press, 1986.

[23] C. Iliadis, Nuclear Physics of Stars, Wiley VCH, 2007.

[24] W. Weinhold, B. Friman and W. Nörenberg, Phys. Lett. B 433 (1998) 236.

[25] R. Garcia-Martin, J. R. Pelaez and F. J. Yndurain, Phys. Rev. D 76 (2007) 074034.

[26] F. Karsch, Prog. Theor. Phys. Suppl. 168 (2007) 237.

[27] Y. Aoki et al., JHEP 06 (2009) 088. 